\title{
HERRAMIENTA MULTIMEDIAL PARA EL ESTUDIO DE LA ANATOMÍA DEL OÍDO A TRAVÉS DE MODELOS VIRTUALES
}

\author{
A MULTIMEDIAL TOOL TO STUDY THE EAR ANATOMY FROM VIRTUAL MODELS
}

\author{
Byron Alfonso, Pérez Gutiérrez \\ Ing. Electrónico, M.Sc., Docente de tiempo completo, Facultad de Ingeniería. \\ Ingeniería Mecatrónica Universidad Militar Nueva Granada, Bogotá, Colombia. \\ E-mail: byron.perez@ieee.org \\ Alvaro Joffre, Uribe Quevedo \\ Ing. En Mecatrónica, M.Sc., Estudiante de Doctorado, Facultad de Ingeniería Mecánica \\ Universidade Estadual de Campinas, Campinas, São Paulo, Brasil. \\ E-mail: engajuq@fem.unicamp.br
}

Fecha de recepción: 27 de mayo de 2009

Fecha de aprobación: 24 de septiembre de 2009

\section{RESUMEN}

Este trabajo presenta la metodología planteada y aplicada para desarrollar una herramienta de tipo multimedia, basada en estándares de internet e imágenes tridimensionales con el objetivo de complementar el proceso de aprendizaje relacionado al estudio de la anatomía del oído humano a través del uso de objetos 3D que incrementen el grado de inmersión e interacción respecto a los modelos reales. Las ayudas visuales tradicionales están basadas en fotografías, ilustraciones, bosquejos y observación directa de los tejidos a través de microscopio, y todas ellas, aun cuando complementarias, presentan algunas limitantes debido a la naturaleza microscópica de algunas partes del oído. Para solucionar las deficiencias presentadas en las técnicas actuales, se propone el desarrollo de una herramienta computacional complementaria que incluya modelos 3D animados e información sobre cada órgano siguiendo los requerimientos de especialistas en el área. Los modelos 3D fueron desarrollados a partir tomografías computarizadas, fotografías y modelos reales, con el fin de obtener un gran realismo y similitud con su contraparte real. La edición de los modelos anatómicos tuvo en cuenta aspectos técnicos de los equipos de cómputo estándar con el fin de garantizar que los usuarios tengan no solo acceso a la herramienta, sino que puedan utilizarla sin una reducción poligonal que afecte el realismo de los modelos y la navegación fluida en el ambiente virtual. La validación de este trabajo fue realizada por especialistas en el 
área, con el objetivo de garantizar que se cumpla con los requerimientos de información y navegación, para poder ser probada en grupos de estudiantes como herramienta complementaria de estudio.

Palabras clave: oído, VRML, multimedia.

\begin{abstract}
This paper brings up a defined and applied methodology for developing a multimedia web-based and 3D image tool. This to supplement the human ear anatomy learning process by using virtual objects in order to enhance the immersion and interaction in relation to real ones. Traditional learning aids are based on photographs, illustrations, sketches and direct tissue observation through microscopes, but although they supplement each other, some limitations rise due to the microscopic nature of some parts, bones and tissues. In order to overcome physical restrictions, a development of a computational tool offering 3D animated and interactive models of the human ear apparatus along with its related information is proposed according to the specifications given by professionals in the area. The 3D models were developed using medical images, such as computerized tomography, photographs and real models, so realistic virtual objects may be created. The editing process of the anatomic models kept in mind the hardware features of standard computers, so a wide range of users may access and use the tool without losing realism. The validation of this work was done by specialists to guarantee the information and navigation requirements fulfillment of the future testing as an alternative educational tool.
\end{abstract}

Keywords: ear, interaction, multimedia.

\title{
INTRODUCCIÓN
}

El siguiente documento presenta la metodología utilizada para desarrollar una herramienta multimedia que permita complementar el material, guías y ayudas visuales disponibles utilizadas en el estudio de la anatomía del oído.

El estudio de diferentes estructuras anatómicas humanas se hace a través de ayudas visuales basadas en fotografías tomadas con cámaras y microscopios digitales [1], maquetas construidas para resaltar a una escala mayor las partes del aparato auditivo, ilustraciones y bosquejos realizados por artistas [2], y en algunos casos dibujos improvisados por el mismo profesor durante el curso. Como complemento a la formación del conocimiento sobre la anatomía del oído, las prácticas con cadáveres han permitido un acercamiento e interacción con la parte u órgano real teniendo en cuenta sus características morfológicas como textura peso y viscosidad. Actualmente los avances en 
computación gráfica a nivel de hardware y software han posibilitado un acercamiento entre la Realidad Virtual (RV) y la medicina [3], permitiendo desarrollar aplicativos para planear cirugías [4] y realizar entrenamiento para el desarrollo de destrezas [5], como parte de la formación y ejecución de procedimientos mínimamente invasivos [6], que tiene por objetivo efectuar intervenciones más efectivas y menos dolorosas.

Con el propósito de hacer más dinámico e interactivo el material de enseñanza basado en fotografías o ilustraciones, en [7] el autor presenta una herramienta para internet que integra imágenes estáticas 2D junto con la información del aparato auditivo. Otro abordaje respecto del problema con los métodos tradicionales se presenta [8], en este trabajo los autores utilizan solamente animaciones sobre el funcionamiento del oído sin ofrecer interactividad con la información o los modelos anatómicos.

Aprovechando las ventajas que ofrecen los sistemas de RV, en $[9,10]$ se presentan trabajos en los cuales se utilizan modelos tridimensionales (3D) que son complementados con información de cada órgano a través de ventanas emergentes. Dicha visualización del contenido interactivo obstaculiza la lectura de la información, creando un ambiente de distracción donde el usuario debe alternar constantemente las ventanas para leer y navegar el modelo.

La ventaja de usar representaciones en tres dimensiones radica en la naturaleza estéreo del sistema visual humano que permite percibir profundidad en la región de estereopsis donde ambos ojos registran la misma imagen [11]. Esta característica es de gran relevancia, ya que el sistema visual humano es un medio sensorial importante en el aprendizaje cotidiano. Estudios realizados permiten apreciar una distinción importante debido al manejo espacial inherente de los ambientes 3D $[12,13]$, ya que el uso de estos facilita el estudio y entendimiento de la anatomía del oído, permitiendo al usuario aproximarse más a las diferentes partes que los métodos tradicionales, alcanzando un grado de inmersión e interacción sin límites y restricciones físicas.

Teniendo en cuenta las ventajas ofrecidas por los ambientes virtuales y la necesidad planteada por los especialistas, se propone el desarrollo de una herramienta complementaria a los métodos tradicionales de enseñanza, en la que el usuario esté inmerso en un ambiente tridimensional generado por computador que contará con información detallada sobre el órgano o parte con la que se esté interactuando.

\section{MATERIALES Y MÉTODOS}

Toda herramienta de RV tiene ciertos elementos sin los cuales no es posible su desarrollo, en [14] se plantean tres componentes esenciales, Imaginación, Interactividad e Inmersión. Cada uno de ellos cumple un objetivo importante, de esta forma la ima- 
ginación de los desarrolladores afectará el desempeño de la herramienta pues una interactividad pobre o un bajo realismo no conseguirán captar la atención del usuario. Tomando la definición de interactividad de [15] como "Conjunto de diálogos y acciones a través de las cuales un usuario humano utiliza e interactúa con un computador", se necesita garantizar un ambiente intuitivo no solamente desde el punto de vista simbólico como formas, texturas, colores, navegación y diálogos, sino también desde el punto de vista no simbólico sacando provecho de la ergonomía del cuerpo humano como propone en su estudio [16], donde se recomienda el uso de dispositivos relacionados al tacto. El rol de la inmersión está relacionado con el estimulo que se realiza sobre los sentidos humanos, debido a que la herramienta propuesta está orientada desde el punto de vista simbólico, resaltando la interactividad a través del nivel de realismo de los modelos presentados teniendo en cuenta aspectos como formas, texturas, detección y manejo de colisiones y tamaños.

Teniendo en cuenta los antecedentes y el propósito de esta herramienta, se plantean los siguientes requerimientos mínimos funcionales:

- Visualización y navegación de los modelos 3D de la anatomía externa, media e interna del oído.

- Accesibilidad de los conceptos teóricos y modelos 3D de forma simultánea para garantizar el foco de concentración del usuario ofreciendo un nivel de interactividad cómodo e intuitivo para el usuario basado en la jerarquía del aparato auditivo.

- Escalabilidad de la herramienta para ser empleada a través de medios como discos compactos, red de área local o red de informática mundial (internet), garantizando portabilidad e interoperabilidad con los estándares de internet y de contenido 3D.

\subsection{INTERFAZ DE USUARIO}

El objetivo principal de la interfaz gráfica del usuario es proveer un ambiente que permita mantenerlo inmerso dentro del mundo virtual junto con la información correspondiente de la parte u órgano que se esté consultado dentro de una misma ventana de la herramienta. Para mantener el foco de atención del usuario se propone una ventana de navegación como se ilustra en la Figura 1, donde la interacción está basada en el uso de dispositivos de interfaz humana, básicos como el ratón (mouse), el teclado y otros avanzados como controles de videojuegos (joysticks), dispositivos haptic ${ }^{1}$ o tablas digitalizadoras.

Dispositivos que usan sensaciones táctiles en la interfaz con el usuario. 


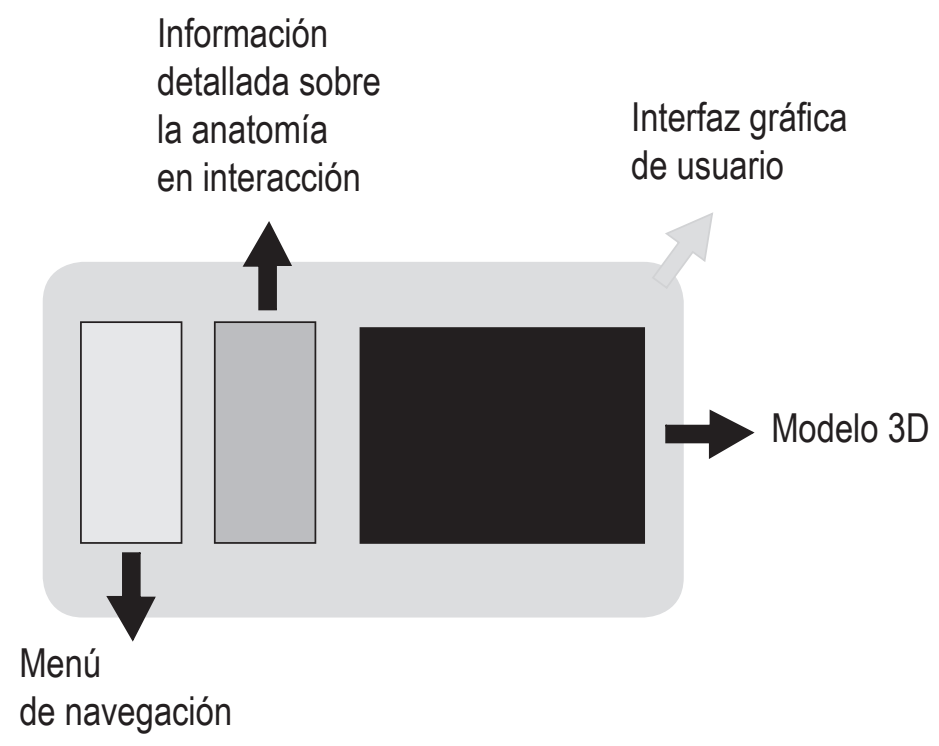

Figura 1. Interfaz gráfica de usuario

La interactividad planteada es de tipo simbólico y se compone de tres áreas, un menú de navegación de tipo desplegable agrupando la información del oído externo, medio e interno, una área con barras de deslizamiento conteniendo la información consultada e hipervínculos al contenido visual y finalmente el modelo 3D con hipervínculos al texto de interés.

Para estructurar el menú de navegación, se toma como base la jerarquía anatómica del oído, de forma que sea intuitiva y clara la presentación del contenido para el usuario, permitiendo ubicar con facilidad y rapidez los datos deseados. Debido a que la información y el material visual relacionado con la anatomía del oído no constituyen el total de una herramienta multimedia, se debe incluir información adicional para llamar la atención y motivar al usuario a utilizar la herramienta. La inclusión de una página inicial donde se reciba cordialmente al usuario y otra donde se introduzcan los objetivos, ventajas y modo de empleo de la herramienta. Los anteriores componentes hacen parte del proceso de ofrecer información que proporcione autonomía al usuario en el momento de utilizar la herramienta. La Figura 2 presenta la estructura utilizada para crear el menú de navegación considerando los componentes de la herramienta. 


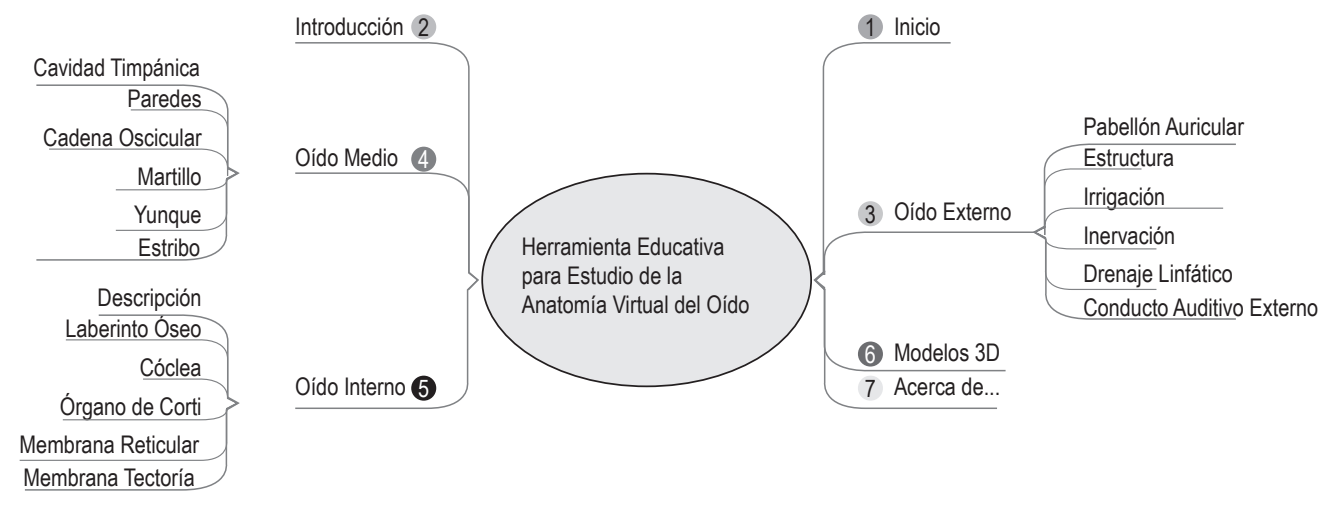

Figura 2. Estructura de navegación de la aplicación

\subsection{CONTENIDO 3D}

Para la creación del contenido virtual, se determina el estándar 3D más adecuado teniendo como limitante de desarrollo la infraestructura de equipos y programas disponibles. Partiendo de la premisa que el realismo ofrecido por los modelos virtuales de la herramienta va a estar enfocado en la forma, tamaño y textura de las partes del oído y no en la simulación avanzada de comportamiento de membranas, órganos y tejidos, así como render ${ }^{2}$ realista, ya que estos últimos afectarían el requerimiento mínimo de interoperabilidad pues necesitarían de sistemas de cómputo y visualización más avanzados, que restringirían el número de usuarios.

Se determinó así, que el lenguaje de modelado de realidad virtual (VRML) [17] ofrece los requisitos necesarios para el desarrollo de contenido interactivo 3D. Tomando en cuenta las características del estándar VRML2.0, se deben tener en cuenta las siguientes consideraciones para el uso de contenido 3D dentro del lenguaje de hipertexto estructurado (HTML) para páginas de internet de forma que se obtengan modelos navegables sin necesidad de equipos especializados: número de polígonos y superficies según se modele de forma poligonal o a través de curvas, calidad de las texturas teniendo en cuenta aspectos como su resolución, número de puntos por pixel (DPI), iluminación y contraste.

2 Proceso que permite generar una imagen a partir de un modelo utilizando el computador. 
Tabla 1. Componentes del aparato auditivo

\begin{tabular}{|c|c|c|c|}
\hline Oído & Externo & Medio & Interno \\
\hline \multirow{4}{*}{ Parte-órgano } & Pabellón & Yunque & Cóclea \\
\cline { 2 - 4 } & Conducto auditivo externo & Martillo & Órgano de Corti \\
\cline { 2 - 4 } & & Estribo & Laberinto óseo \\
\cline { 2 - 4 } & & Membrana timpánica & Membrana reticular \\
\cline { 2 - 4 } & & \multicolumn{2}{|c|}{ Membrana tectoria } \\
\cline { 2 - 4 } & \multicolumn{3}{|c|}{ Hueso temporal } \\
\hline
\end{tabular}

Teniendo claros los parámetros de desarrollo, la construcción de los modelos 3D inicia con la definición de los órganos y partes de interés (tabla 1), así, para el modelado de cada uno de ellos se tomó como referencia el material disponible en la literatura especializada [18, 19, 20], maquetas e imágenes de tomografías computarizadas de las zonas de interés siguiendo el proceso descrito en la Figura 3.

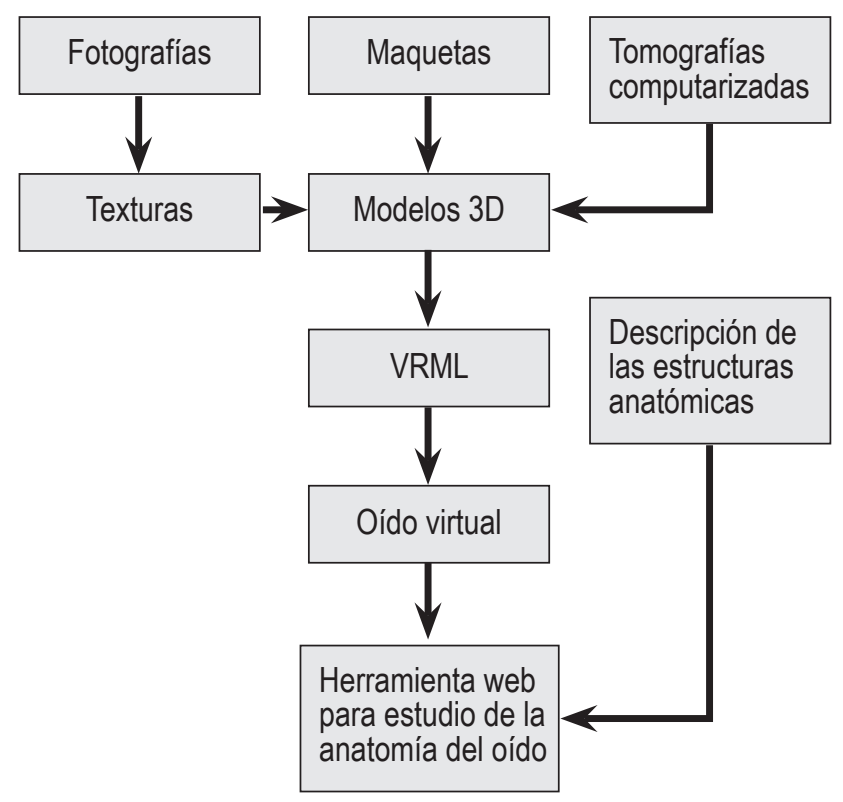

Figura 3. Proceso de construcción de la herramienta propuesta

El proceso de modelado es integral ya que las tres fuentes de información se complementan permitiendo conseguir mayor detalle y precisión en los modelos. Las tomografías computarizadas permiten la creación de modelos 3D basados en modelos reales, las imágenes y fotografías permiten la creación de texturas apropiadas para conseguir rea- 
lismo y finalmente las maquetas y huesos permiten obtener mayor detalle e información adicional para garantizar una navegación similar a la real dentro del aparato auditivo.

La construcción del oído externo y medio se basó en un modelo creado a partir de una pila de imágenes de tomografía computarizada de la cabeza humana haciendo uso del algoritmo marching cubes [21]. Debido al fenómeno de aliasing, en el cual las imágenes presentan artefactos representados por bordes muy escalonados, se aplicó un filtro pasa-bajos a la imagen original y se obtuvo un modelo del que se puede reconstruir la geometría de interés (Figura 4).
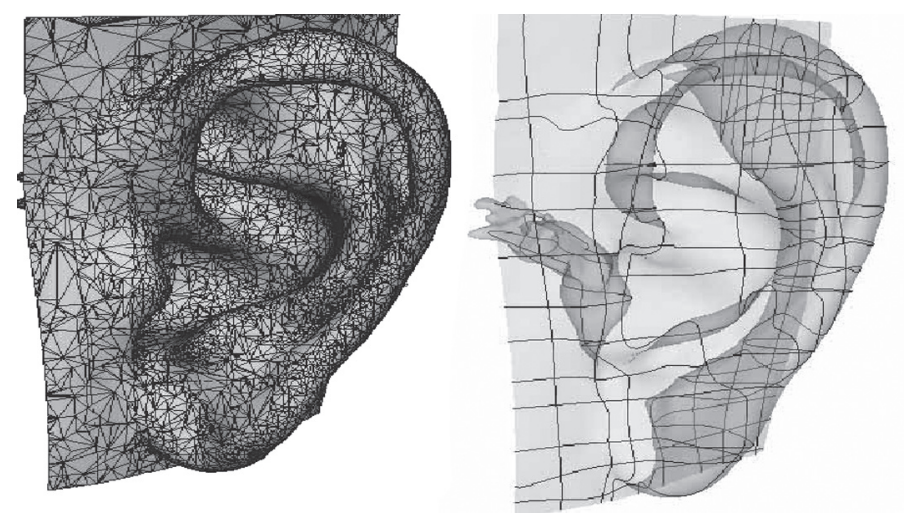

Figura 4. Malla poligonal (izquierda) y curvas principales (derecha) del pabellón auricular reconstruido

Debido a los tamaños de los órganos del oído medio e interno, su modelado 3D no fue posible a partir del procesamiento de imágenes médicas resultantes de tomografías o resonancias. Para la creación de cada componente del oído medio e interno, se utilizaron imágenes y fotografías tomadas desde diferentes ángulos por un especialista haciendo uso de un microscopio. La anterior información visual aportó suficientes datos sobre la forma y tamaño de cada parte para realizar con base en ellas la reconstrucción virtual de cada órgano. La Figura 5 permite visualizar los resultados obtenidos para la cóclea y el órgano de Corti.

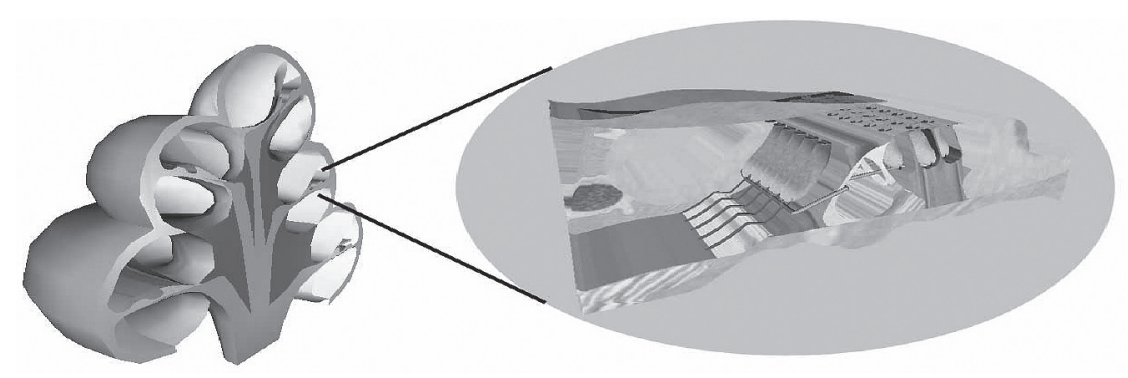

Figura 5. Cóclea y detalle del órgano de Corti 
Una de las funciones importantes en el proceso auditivo la cumple la cadena oscicular (Figura 6), ya que al recibir las ondas sonoras las transmite a la membrana timpánica. Para permitir que el usuario disponga de una representación espacial de cómo transmite esta información, se realizó una animación que representa la reacción de la cadena oscicular ante un estímulo mecánico.

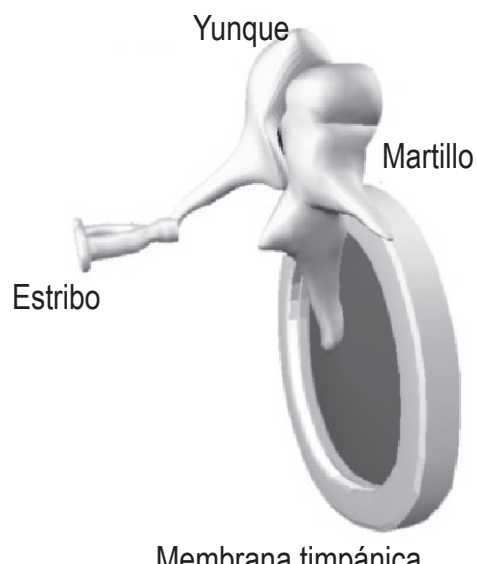

Figura 6. Cadena oscicular animada

Una vez todos los componentes están modelados, estos se integran dentro del hueso temporal junto con el canal auditivo y el pabellón externo. Durante esta etapa se realiza la edición de los modelos VRML, donde se especifican las propiedades de las cámaras (campo de visión, tamaño y posición), luces (intensidad, color, tipo y posición) y texturas (mapeo en las tres dimensiones) que permiten la obtención del modelo presentado en la Figura 7.

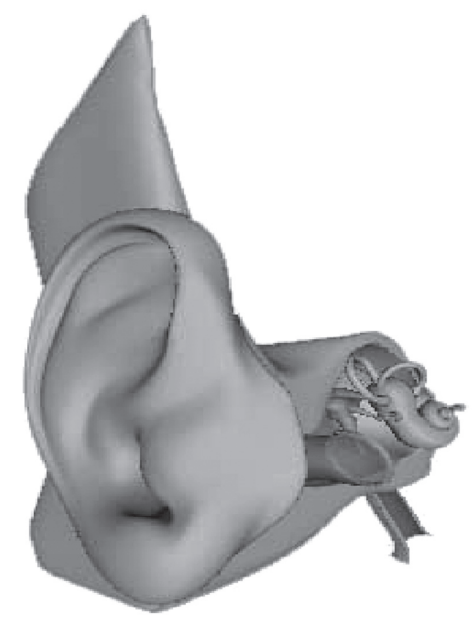

Figura 7. Modelo del oído en VRML 
Los aspectos de navegación como tamaño del observador virtual y desplazamiento, colisiones, e interacción con dispositivos de interfaz humana también se configuran en la etapa de edición, así por ejemplo, se define la interacción a través del ratón (ya que este es un dispositivo de interfaz humana de bajo costo y uso común) de la siguiente forma: el botón derecho permite realizar desplazamientos horizontales y verticales, el botón medio permite rotar, y el botón izquierdo permite al usuario acercarse o alejarse del objeto.

\section{RESULTADOS Y ANÁLISIS}

Como resultado de seguir la metodología propuesta se consiguió realizar la implementación y desarrollo de una herramienta tipo multimedia compuesta por modelos 3D de la anatomía del oído, información teórica relacionada con cada parte de este órgano, y finalmente soportada por el estándar HTML para su uso con o sin conexión a internet. El uso de ambientes 3D, imágenes en conjunto con texto plano e hipertexto, permitió la obtención de una herramienta dinámica, intuitiva e interactiva, que complementa los métodos tradicionales de enseñanza.

La diagramación de la interfaz gráfica de usuario, que está basada en la estructura anatómica del oído, ofrece un árbol de navegación intuitivo que permite el acceso fácil y rápido de la información, permitiendo mantener el foco de concentración en la lectura y los modelos 3D. Las anteriores características posibilitaron una buena inmersión simbólica, pues en conjunto con colores, texturas y formas, la herramienta mantiene al usuario interesado sin distraerlo, como se presenta en la Figura 8.

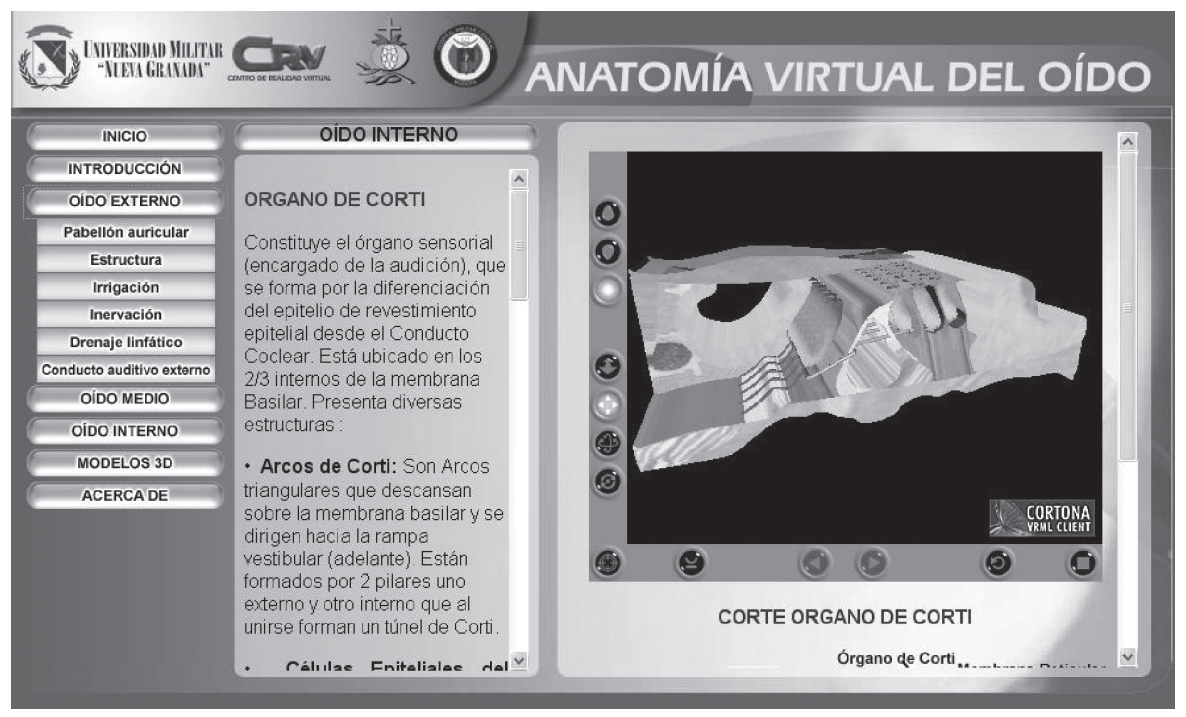

Figura 8. Interfaz gráfica de usuario 
Un grupo de 3 expertos en el área validaron los modelos y la interfaz gráfica, encontrando que con el modelo más detallado de todo el oído (figura 7) no fue posible realizar rotaciones y traslaciones en forma interactiva y fluída, debido a las características del computador que se tenia disponible. En cuanto a la forma, color y textura de los modelos no tuvieron observaciones y los encontraron adecuados para ser integrados en el proceso de aprendizaje de la anatomía del oído.

Teniendo en cuenta estas observaciones se hicieron pruebas del rendimiento de la aplicación a nivel de velocidad de cuadros por segundo (frames per second - FPS) con dos configuraciones de computador (tabla 2). Las pruebas consistieron en cargar un modelo de todo el oído (externo, medio e interno simultáneamente de 21614 vértices, ver figura 7) y comparar el número de FPS en tres escenarios: estático, con rotación y con acercamiento, en los dos computadores.

Tabla 2. Características técnicas de los computadores utilizados en las pruebas

\begin{tabular}{|l|l|l|}
\cline { 2 - 3 } \multicolumn{1}{c|}{} & \multicolumn{1}{c|}{ Computador 1 } & \multicolumn{1}{c|}{ Computador 2 } \\
\hline Memoria RAM & 4GB & 256MB \\
\hline Memoria de video & $512 \mathrm{MB}$ (Tarjeta de video independiente) & $\begin{array}{l}16 \mathrm{MB} \text { (Tarjeta de video integrada a } \\
\text { la placa madre) }\end{array}$ \\
\hline Procesador & Intel Core Duo de $2 \mathrm{GHz}$ & Intel Pentium III de $800 \mathrm{MHz}$ \\
\hline
\end{tabular}

Los resultados mostraron un resultado de 80 FPS en el computador 1 vs 5 FPS en el computador 2. Esta diferencia tan grande se debe a que la tarjeta de video independiente tiene un desempeño superior a las que vienen integradas en la placa madre, sin embargo, para mejorar el desempeño en el computador 2 , se realizó una reducción de la malla poligonal mediante el proceso de decimado [22] en los modelos 3D más complejos y se redujo el tamaño de las texturas usadas. La figura 9 muestra los modelos reducidos.

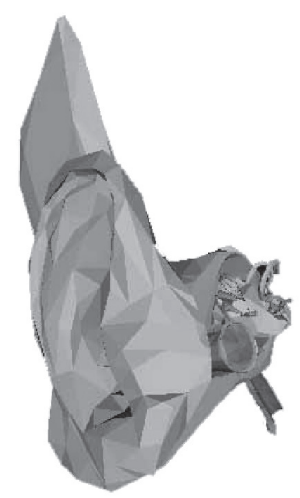

a) Vértices $=2018$

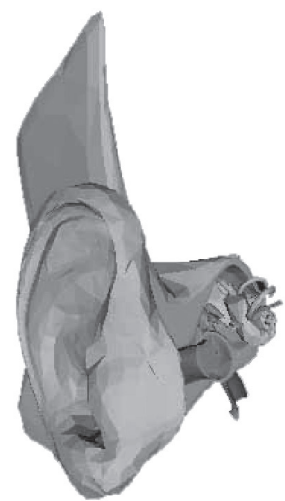

b) Vértices $=7589$

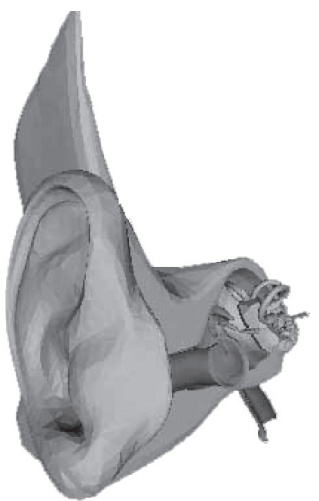

c) Vértices $=12859$

Figura 9. Geometrías obtenidas a partir del decimado del modelo original de la figura 7. 
La tabla 3 muestra los resultados de las pruebas realizadas sobre los tres modelos reducidos y el original. Se puede observar que entre menor número de vértices, mayor es el número de FPS obtenido en el computador 2, sin embargo con la reducción a 2018 vértices la calidad del modelo disminuye mucho y se pierde una de las características deseables que es el render realista. El modelo de 12859 vértices es el que presenta el mejor desempeño global al tener una aceptable calidad de imagen combinada con el rango de 9.8 a 11 FPS en movimiento, siendo estos valores los mínimos para que la aplicación sea interactiva y el usuario no tenga que esperar un tiempo significativo desde que realiza el movimiento de la geometría y se visualiza su resultado en la pantalla.

Tabla 3. Resultados con tres niveles de reducción de la malla comparados con la geometría original

\begin{tabular}{|c|c|c|c|c|c|c|c|c|}
\hline Computador & 1 & 2 & 1 & 2 & 1 & 2 & 1 & 2 \\
\hline Vértices & \multicolumn{2}{|c|}{ a) 2018} & \multicolumn{2}{|c|}{ b) 7589} & \multicolumn{2}{|c|}{ c) 12859} & \multicolumn{2}{|c|}{ Original 21614} \\
\hline FPS estático & 83 & 81 & 83 & 81 & 83 & 81 & 83 & 81 \\
\hline FPS rotando & 60 & 29 & 60 & 14.7 & 60 & 9.8 & 30 & 11 \\
\hline FPS acercando & 60 & 19 & 60 & 14 & 60 & 11 & 60 & 9 \\
\hline Tamaño en KB & \multicolumn{2}{|c|}{139} & \multicolumn{2}{|c|}{511} & \multicolumn{2}{|c|}{889} & \multicolumn{2}{|c|}{3222} \\
\hline
\end{tabular}

La última prueba realizada consistió en acceder la herramienta a través de Internet tomando como parámetro de medición el tiempo de carga de los modelos. Los resultados mostraron que al utilizar el modelo original se tiene requiere de un mayor tiempo de descarga (25.7s con una conexión de 1Mbps) sin embargo el estándar VRML permite manejar versiones comprimidas mediante GZIP (http://www.gzip.org), logrando reducir el tamaño a $637,5 \mathrm{~KB}$ y el tiempo de carga a $5,1 \mathrm{~s}$.

\section{CONCLUSIONES}

En este trabajo se presentó la metodología utilizada para el desarrollo de una herramienta sobre Internet que permite al usuario complementar el proceso de aprendizaje relacionado con el estudio de la anatomía del oído humano.

Las técnicas de modelado empleadas permiten la creación de objetos 3D con alto grado de realismo y un bajo número de polígonos necesarios para no afectar significativamente el desempeño de la herramienta si la tarjeta de video no es capaz de procesar la cantidad de triángulos por unidad de tiempo requerida.

Pruebas realizadas en dos computadores (uno de alto y otro de bajo desempeño), permitieron establecer las características de los modelos 3D para garantizar sufi- 
ciente realismo y fluidez en la navegación. Al combinar lo anterior con el ambiente gráfico y la información respectiva de cada órgano, se obtuvo una herramienta intuitiva e interactiva que permite ser utilizada en un amplio rango de hardware sin que sus características de uso se vean alteradas.

La reducción de polígonos sobre geometrías ya creadas, trae como consecuencia la pérdida de realismo y similitud con sus contrapartes orgánicas. La reducción realizada en diferentes medidas, permite ver un decremento en el número de caras y vértices, lo que también termina afectando las texturas aplicadas. Debido a lo anterior, toda geometría 3D modelada, debe desde un inicio proyectarse para tener el menor número de polígonos, y de esta forma evitar reducciones innecesarias que comprometan el objetivo de la herramienta.

La metodología propuesta, permite no sólo la aplicación al estudio de la anatomía del oído externo, medio e interno, también puede extenderse a otras partes del cuerpo humano donde se relacionen no solo características fisiológicas sino también patológicas, como es el caso del ojo humano, donde algunos diagnósticos como el fondo de ojo requieren entrenamiento para detectar problemas. A través de modelos virtuales, las patologías pueden ser implementadas y presentadas a los estudiantes de forma muy similar a la real como se muestra en la Figura 10.

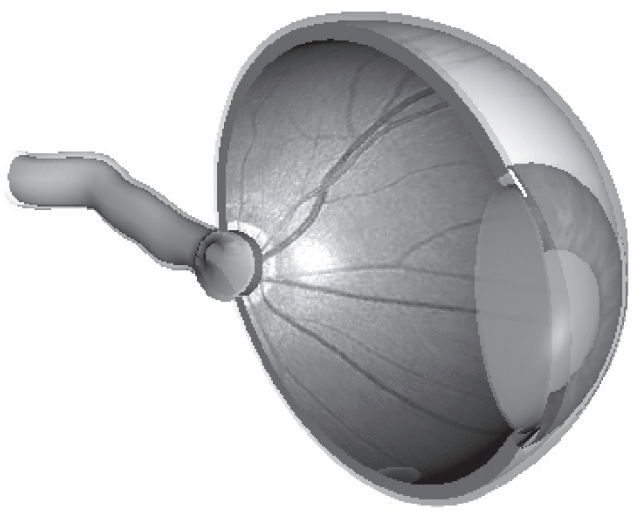

Figura 10. Modelo 3D del ojo con un fondo de ojo normal

La validación de la herramienta por parte de los especialistas, permite tener la seguridad de que, tanto el contenido como los modelos 3D están dentro de las expectativas y necesidades de una solución complementaria a los métodos tradicionales de enseñanza relacionados con la anatomía del oído.

Como trabajo futuro se propone la creación de un módulo de evaluación sobre los conocimientos adquiridos con la herramienta y explorar algoritmos de reconstrucción a 
partir de imágenes de microscopía del oído interno. También se propone mantener la herramienta actualizada para mantener su portabilidad con las tendencias en navegadores web y sistemas operativos.

\section{AGRADECIMIENTOS}

El presente trabajo se realizó con el apoyo de la especialización en otorrinolaringología de la Facultad de Medicina - Universidad Militar Nueva Granada, Hospital Militar Central y Hospital Universitario Clínica San Rafael, Dr. Jorge Eduardo Almario, Dr. José Alberto Prieto y Dra. Martha Liliana Bretón.

\section{BIBLIOGRAFÍA}

[1] Johannes W. Rohen, Chihiro Yokochi, Elke Lütjen-Drecoll. Color Atlas of Anatomy, Fourth Edition.

[2] Eliot Goldfinger, Human Anatomy for Artists: The Elements of Form, lustrated edition, Noviembre 7, 1991.

[3] Waterworth, J. A. (1999), 'Survey of Medical Applications of Virtual Reality'(9802), Technical report, Dept of Informatics Umea University. http://www.informatik.umu.se/ jwworth/medpage.html.

[4] B. Reitinger, D. Schmalstieg, A. Bornik, R. Beichel Spatial Analysis Tools for Virtual Reality-based Surgical Planning In Proc. of the IEEE Symposium on 3D User Interfaces 2006, 2006.

[5] Papadopoulos, E.; Tsamis, A. \& Vlachos, K. (2008), 'Development of a realtime visual and force environment for a haptic medical training simulator', Artif Life Robotics 12, 307-316.

[6] Stoyanov, D.; ElHelw, M.; Lo, B. P.; Chung, A.; Bello, F. \& Yang, G.-Z. (2003), Current Issues of Photorealistic Rendering for Virtual and Augmented Reality in Minimally Invasive Surgery, in 'Seventh International Conference on Information Visualization (IV'03) July 16 - 18, London, England'.

[7] Perry Hanavan. Virtual tour of the ear. Augustana College. http://www.augie.edu/perry/ear/ear.htm

[8] J. Brugge, T. Pasic, B. Rhode, T. Yin. EarWorks - Hearing and balance. University of Wisconsin - Madison. 
[9] 3D ear an anatomy tutorial.

http://audilab.bmed.mcgill.ca/ daren/3Dear/3d models.html

[10] Gross anatomy of the ear. http://sprojects.mmi.mcgill.ca/oto3d/

[11] Arditi, A. (1986) Binocular Vision. In The Handbook of Perception and Human Performance, Volume I: Sensory Processes and Perception, Boff, Kaufman \& Thomas (Eds.), Wiley:NY.

[12] Daren T Nicholson, Colin Chalk, W Robert J Funnell, Sam J Daniel. Can virtual reality improve anatomy education? A randomised controlled study of a computer-generated three-dimensional anatomical ear model. Medical Education 2006. N.40, pp.1081-1087. Blackwell Publishing 2006.

[13] Sánchez A., Barreiro J, Maojo V, "Design of Virtual Reality Systems for Education: A Cognitive Approach". Education and Information Technologies, Vol 5 N(4), p.p. 345-362 2000.

[14] Burdea G., Coiffet P., "Virtual Reality Technology”, Wiley-IEEE Press; 2 Edition, June 2003.

[15] Baecker R.,Buxton W. "Readings in human-computer interaction: A multidisciplinary approach”. San Mateo, CA: Morgan Kaufmann Publishers, 1987.

[16] Crawford C., "Understanding interactivity", 2000.

[17] Virtual Reality Modeling Language (VRML97) ISO/IEC 14772:1997. http://www.web3d.org/x3d/specifications/

[18] Johannes W. Rohen, Chihiro Yokochi, Elke Lütjen-Drecoll. Color Atlas of Anatomy, Fourth Edition.

[19] Testut L., Latarjet A. Tratado de Anatomía Humana. Salvat Editores. Tomo 3. 1978.

[20] Rohen, Yokochi. Atlas fotográfico de anatomía humana. Doyma. 2da Edición. 1987.

[21] Lorensen, W., Cline, H. Marching cubes: A high resolution 3D surface construction algorithm Computer Graphics - Proc. SIG-GRAPH 87, 1987, Vol 21 No.(4), 163-169.

[22] Schroeder, W. J.; Zarge, J. A. \& Lorensen, W. E. (1992). Decimation of Triangle Meshes. Proc. ACM-Siggraph, 92. 